\title{
Decreased expression of miR-204 in plasma is associated with a poor prognosis in patients with non-small cell lung cancer
}

\author{
WEIGANG GUO, YONGXING ZHANG, YI ZHANG, YU SHI, JUNJIE XI, HONG FAN and SONGTAO XU \\ Department of Thoracic Surgery, Zhongshan Hospital of Fudan University, Shanghai 200032, P.R. China
}

Received March 28, 2015; Accepted October 13, 2015

DOI: $10.3892 / \mathrm{ijmm} .2015 .2388$

\begin{abstract}
In order to identify novel non-invasive biomarkers with high accuracy for the screening of non-small cell lung cancer (NSCLC), we investigated the predictive power of 4 microRNAs (miR-146, miR-204, miR-106a and miR-124) in plasma samples obtained from patients with NSCLC and healthy controls $(n=50$; training phase) by reverse transcription-quantitative polymerase chain reaction (RT-qPCR). We found that the levels of miR-204 in the patients with NSCLC were significantly dysregulated compared with the healthy controls, and thus this miRNA was selected for further validation. For the validation phase, RT-qPCR was performed on plasma samples from patients with NSCLC and healthy controls $(n=176)$ in order to examine the expression levels of the candidate miRNA, miR-204. The results revealed that the plasma levels of miR-204 were significantly downregulated in the patients with NSCLC compared with the healthy controls $(\mathrm{p}<0.001)$. The value of the area under the receiver operating characteristic (ROC) curve obtained for miR-204 was 0.809 (sensitivity, $76 \%$; specificity, $82 \%$ ), which was higher than the values obtained for carcinoembryonic antigen (CEA) and carbohydrate antigen 19-9 (CA19-9). The expression of miR-204 in plasma significantly correlated with the tumor stage $(\mathrm{p}=0.009)$ and distant metastasis $(\mathrm{p}=0.048)$. A log-rank test revealed that lower plasma levels of miR-204 were associated with a shorter overall survival and disease-free survival $(p=0.006$ and 0.0065 , respectively). Both univariate and multivariate Cox regression analyses indicated that a lower miR-204 expression level in plasma was a prognostic factor with a relative risk of death of 1.936 and 1.712 , respectively. On the whole, our results suggest that the decreased expression of miR-204 in plasma is a promising biomarker for the detection of NSCLC and the prediction of poor survival in patients with the disease.
\end{abstract}

Correspondence to: Professor Songtao Xu, Department of Thoracic Surgery, Zhongshan Hospital of Fudan University, 180 Fenglin Road, Shanghai 200032, P.R. China

E-mail: songtxu001@163.com

Key words: miR-204, non-small cell lung cancer, plasma, diagnosis, prognosis

\section{Introduction}

Lung cancer still remains the most common type of cancer and the leading cause of cancer-related mortality worldwide, particularly in China $(1,2)$. Although there have been recent advances in surgical resection and chemoradiation therapy, the prognosis for patients with lung cancer, particularly non-small cell lung cancer (NSCLC), remains poor, with a 5-year survival rate of no more than $15 \%$, which has not improved over the past years (3). The lack of an effective biomarker for the early screening and detection of NSCLC is the reason for that the majority of NSCLC cases are diagnosed at advanced stages, which leads directly to a high mortality rate in patients with this type of cancer. Therefore, the identification of non-invasive biomarkers is urgently required to complement and advance current diagnostic and prognostic tools for NSCLC.

MicroRNAs (miRNAs or miRs) are endogenously expressed non-coding RNA molecules that cause mRNA translation, inhibition or degradation via direct base-pairing interactions, which mainly repress the expression of several putative targets post-transcriptionally (4). Moreover, previous research has demonstrated that miRNAs are abnormally expressed in various types of cancer, and act as oncogenes or tumor-suppressor genes to regulate a wide range of biological processes, such as tumorigenesis, differentiation, proliferation, apoptosis, tumor invasion and metastasis $(5,6)$. The dysregulation of specific miRNAs can also serve as an effective biomarker for predicting prognosis in patients with various types of cancer, including NSCLC (7-9). More importantly, certain studies have indicated that circulating miRNAs are stable enough to be detected in serum and plasma $(10,11)$, and thus may be promising non-invasive biomarkers for the early detection and prognosis of cancers. Indeed, previous studies have demonstrated that miRNAs in plasma may be utilized as novel diagnostic or prognostic biomarkers in various types of cancer, including colorectal cancer (12), prostate cancer (13), breast cancer (14) and gastric cancer (15).

Of these aberrantly expressed miRNAs, 4 common miRNAs (miR-124, miR-204, miR-146 and miR-106a) have been shown to be associated with tumor-related biological processes, and are dysregulated in patients with cancer, compared to healthy individuals. miR-204 is located at chromosome 9q21.12, and recent studies have found that it is downregulated in gastric cancer (16), breast cancer (17), renal cell carcinoma (18), and NSCLC (19). Functionally, the overexpression of miR-204 in cancer cells inhibits migration and proliferation, and promotes 
apoptosis (20-23). Moreover, it has also been reported that miR-124, miR-146 and miR-106a act as oncogenic or tumorsuppressor genes which are involved in tumor progression. However,to the best of our knowledge, no previous research to date has focused on the correlation between the expression levels of miR-124, miR-204, miR-146 and miR-106a in plasma and NSCLC. Therefore, we conducted this study to evaluate whether these 4 miRNAs could serve as useful biomarkers in the screening of NSCLC.

For the purposes of our study, we examined previous studies and found that the plasma levels of 4 miRNAs (miR-124, miR-146, miR-204 and miR-106a) appear not to have been studied in relation to NSCLC. Thus, we first measured the expression levels of these miRNAs in plasma samples from patients with NSCLC (training phase). Of these tested miRNAs, only miR-204 was found to be dysregulated in the patients with NSCLC, and thus it was selected for further analysis. In the validation phase, we evaluated the expression levels of miR-204 in patients with NSCLC $(n=126)$ and healthy controls $(n=50)$ to determine whether a correlation exists between its expression and the clinicopathological parameters of patients with NSCLC. We also compared the diagnostic sensitivity and specificity of plasma miR-204 levels with those of conventional NSCLC biomarkers, such as carbohydrate antigen 19-9 (CA19-9) and carcinoembryonic antigen (CEA) in blood.

\section{Subjects and methods}

Subjects and tissue samples. The study population consisted of 151 plasma samples obtained from patients with NSCLC who underwent primary surgical resection at the Department of Thoracic Surgery, Zhongshan Hospital of Fudan University (Shanghai, China), between January 2009 and May 2011. We obtained blood samples from patients prior to surgery. In addition, 15 cases were randomly selected for collecting blood samples post-operatively. Patients who had received pre-operative chemo- or radiotherapy were excluded from this study. Clinical information on histological type, tumor stage and lymph node involvement was obtained from medical records. Patients who had stopped smoking for over 2 years were defined as former smokers. All patients were regularly followed-up post-surgery though clinical visits or through telephone communications. Two surgical pathologists performed histological evaluations of the tumors. All cases of cancer were staged according to the guidelines of the tumor- nodemetastasis (TNM) classification of the Union for International Cancer Control (UICC). A total of 75 age- and gender-matched healthy controls were also selected, including smokers and non-smokers, but with no history of tumors.

This study was approved by the Ethics Committee of Zhongshan Hospital of Fudan University. All patients provided written informed consent indicating their willingness to donate their blood for scientific research.

Study design. This study was divided into 2 phases, the training phase and validation phase. In the training phase, we selected 25 patients with early-stage NSCLC and 25 age- and gendermatched healthy controls to compare the expression profiles of these 4 miRNAs between patients with NSCLC and healthy individuals. According to the training set results, we found that some of the miRNAs exhibited statistically significant differences in expression, and we thus conducted a validation experiment to further examine the diagnostic and prognostic performance of these miRNAs. In the validation set, plasma samples were collected from 126 patients with NSCLC and 50 healthy controls.

Plasma preparation and RNA extraction. Plasma samples were obtained by centrifugation of the peripheral blood $(3 \mathrm{ml}$, collected in EDTA-K2 anti-coagulant tubes) at 3,000 rpm for $20 \mathrm{~min}$ and at $12,000 \mathrm{rpm}$ for $10 \mathrm{~min}$ at $4^{\circ} \mathrm{C}$. The plasma samples were aliquoted and stored in fresh tubes at $-80^{\circ} \mathrm{C}$ for the further analysis.

Total RNA (including miRNAs) from plasma samples was extracted using the miRNeasy mini kit (Qiagen Inc., Valencia, CA, USA) according to the manufacturer's instructions, and dissolved in $20 \mu \mathrm{l}$ RNase-free water. The concentration and quality of the isolated RNA were assessed on a NanoDrop ND-1000 Spectrophotometer (NanoDrop Products, Wilmington, DE, USA). RNA samples were stored at $-80^{\circ} \mathrm{C}$ until further use.

Reverse transcription-quantitative polymerase chain reaction $(R T-q P C R)$. The reverse transcription reaction was conducted using a TaqMan microRNA Reverse Transcription kit (Applied Biosystems, Foster City, CA, USA) in a $15 \mu 1$ solution consisting of $5 \mu \mathrm{l}$ RNA template, $1.5 \mu 1$ 10X reverse transcription buffer, $1 \mu \mathrm{l}$ MultiScribe reverse transcriptase, $0.15 \mu \mathrm{l}$ of $100 \mathrm{mM}$ dNTPs, $0.19 \mu \mathrm{l}$ of RNase inhibitor $(20 \mathrm{U} / \mu \mathrm{l})$, $1 \mu \mathrm{l}$ of gene-specific primer, and supplemented with nucleasefree water. For the synthesis of complementary DNA (cDNA), the reaction mixtures were incubated at $16^{\circ} \mathrm{C}$ for $30 \mathrm{~min}$, at $42^{\circ} \mathrm{C}$ for $30 \mathrm{~min}$, and at $85^{\circ} \mathrm{C}$ for $15 \mathrm{~min}$ and then held at $4^{\circ} \mathrm{C}$.

RT-qPCR was used to quantify the expression levels of the miRNAs. Each amplification reaction was carried out in a final volume of $20 \mu \mathrm{l}$ containing $2 \mu \mathrm{l}$ of the cDNA (100 ng), $10 \mu$ l of TaqMan 2X Universal PCR Master Mix with No AmpErase UNG (Applied Biosystems), $1 \mu 1$ of gene-specific primer, and $7 \mu \mathrm{l}$ of nuclease-free water. The ABI Prism 7300 RT-qPCR system (Applied Biosystems) was used to detect the plasma miRNA levels. The PCR conditions consisted of an initial polymerase activation step at $95^{\circ} \mathrm{C}$ for $10 \mathrm{~min}, 46$ cycles of $95^{\circ} \mathrm{C}$ for $15 \mathrm{sec}$ and $60^{\circ} \mathrm{C}$ for $1 \mathrm{~min}$. Each reaction was performed in triplicate. The expression levels of the miRNAs in plasma were normalized to the levels of small nuclear RNU6B (U6) and were calculated using the $2^{-\Delta \Delta \mathrm{Ct}}$ method, as previously described (24).

Measurement of CA19-9 and carcinoembryonic levels in serum. Serum levels of CA19-9 and CEA are generally used in the diagnosis of many types of cancer, including NSCLC (25). In this study, serum CA19-9 and CEA levels in patients with NSCLC and healthy individuals were measured by chemiluminescent enzyme immunoassays (Fujirebio, Inc., Tokyo, Japan) according to the manufacturer's instructions. In brief, $50 \mu \mathrm{l}$ of antigen and $50 \mu \mathrm{l}$ of antibody were added to the microwell and thoroughly mixed for $30 \mathrm{sec}$. Subsequently, 5 washings were performed, after which $50 \mu \mathrm{l}$ of chemiluminescence substrate were added, and the mixture was incubated at room temperature in the dark. 
Statistical analysis. The SPSS 16.0 software package (SPSS Inc., Chicago, IL, USA) and GraphPad Prism 5.0 statistical software (GraphPad Software Inc., La Jolla, CA, USA) were utilized for all statistical analyses. The expression levels of miRNAs were calculated based on the $2^{-\Delta \Delta C t}$ method, and were analyzed as the means \pm standard deviation (SD). The independent two-sample Student's t-test was used to analyze the correlations between miR-204 expression and the clinicopathological variables of the patients. The KruskalWallis test was performed to compare the expression of miR-204 in different types of lung cancer. Receiver-operating characteristic (ROC) curves of miR-204, CA19-9 and CEA were generated to differentiate the patients with NSCLC from the healthy controls. The optimal cut-off value for diagnosis was obtained using Youden's Index (sensitivity + specificity -1 is maximal), as previously described (26). The Kaplan-Meier method was used for survival analysis, and the differences in survival curves were compared using the log-rank test. Cox regression analysis was used to estimate the univariate and multivariate hazard ratios for prognosis. All p-values shown are two-sided, and a p-value $<0.05$ was considered to indicate a statistically significant difference.

\section{Results}

Expression of miRNAs in the training set. Of the 25 patients with NSCLC in the training set, 8 patients had stage I and 17 had stage II NSCLC, 12 had adenocarcinoma, 8 had squamous cell carcinoma and 5 had another subtype of NSCLC (Table I). A total of 25 healthy individuals was selected as the normal controls. The age and gender of the healthy controls were matched with those of the patients with NSCLC. Firstly, we measured the expression levels of miR-124, miR-146, miR-106a and miR-204 in plasma from 25 patients with NSCLC and 25 healthy controls. We observed a significant difference in the levels of miR-204 in plasma between the patients with NSCLC and the healthy controls $(0.018 \pm 0.008$ in tumors vs. $0.057 \pm 0.034$ in controls, $\mathrm{p}<0.001)$, whereas the difference in the expression levels of miR-146 $(0.037 \pm 0.029$ in tumors vs. $0.026 \pm 0.013$ in controls), miR-106a $(0.064 \pm 0.045$ in tumors vs. $0.055 \pm 0.039$ in controls) and miR-124 $(0.014 \pm 0.010$ in tumors vs. $0.019 \pm 0.013$ in controls) in plasma between the patients with NSCLC and the controls were not deemed statistically significant (Fig. 1). Thus, we selected miR-204 for further analysis.

Diagnostic performance of plasma miR-204 for NSCLC. In the validation set, the plasma levels of miR-204 in 126 patients with NSCLC and 50 healthy controls (patient characteristics in the validation set are shown in Table II) were measured by RT-qPCR. The expression levels of miR-204 in plasma were significantly decreased in the patients with NSCLC compared to the healthy controls $(0.016 \pm 0.013$ vs. $0.051 \pm 0.041$, $\mathrm{p}<0.001$ ) (Fig. 2A). In addition, as shown in Fig. 2B, the plasma levels of miR-204 in the patients with stage IV NSCLC were much lower than those in the patients with stage I NSCLC ( $\mathrm{p}=0.011)$, whereas no significant differences were observed between the patients with stage II and IV of the disease, or between those with stage III and IV of the disease ( $p>0.05)$. Moreover, the expression levels of miR-204 in plasma were found to be re-decreased in 8 patients who
Table I. Characteristics of patients in training set.

\begin{tabular}{lc}
\hline Characteristics & No. of patients $(\mathrm{n}=25)$ \\
\hline Gender & 16 \\
Male & 9 \\
Female & \\
Age (years) & 10 \\
$<60$ & 15 \\
$\geq 60$ & \\
Smoking history & 9 \\
Never, former & 16 \\
Current & \\
Histological type & 12 \\
Adenocarcinoma & 8 \\
Squamous cell carcinoma & 5 \\
Others & \\
TNM staging & 8 \\
I & 17 \\
II & \\
Lymph node metastasis & 9 \\
No & 16 \\
Yes &
\end{tabular}

Smoking history: former, stopped smoking for $>2$ years; never, no smoking history; current, current smoker.

developed post-operative recurrence of distant metastasis during follow-up examinations (Fig. 2C).

In order to evaluate the diagnostic capabilities of the plasma levels of miR-204, and serum levels of CEA and CA19-9 for NSCLC, ROC curve analysis was performed. The optimal cut-off value for plasma miR-204 levels in the patients with NSCLC was 0.023 , which provided a sensitivity of $76 \%$ and a specificity of $82 \%$, with an area under the curve (AUC) value of 0.809 , which was used for differentiating the patients with NSCLC from the healthy controls. Two conventional NSCLC markers, CEA and CA19-9, were also measured in all subjects. At a cut-off value of 5.6 for the CEA expression levels, the optimal sensitivity and specificity were 70 and $72 \%$, respectively. At a cut-off value of 51.9 for the CA19-9 expression levels, the optimal sensitivity and specificity were 57 and $84 \%$, respectively. The AUC for the plasma level of plasma miR-204 was significantly larger than that for CEA (0.724) or CA19-9 (0.686) (Fig. 3), indicating that the plasma miR-204 levels are superior to the serum levels of CEA or CA19-9 for the detection of NSCLC.

Correlation between plasma miR-204 and clinicopathological characteristics. We evaluated the correlation of plasma miR-204 with clinicopathological parameters, including gender, age, TNM stage and histological type. These data are summarized in Table II. The low level of plasma miR-204 was found to significantly correlated with TNM stage $(\mathrm{p}=0.009)$ and distant metastasis $(\mathrm{p}=0.048)$, whereas no significant correlation between miR-204 and gender, age, differentiation, histological type and lymph node involvement was observed. 

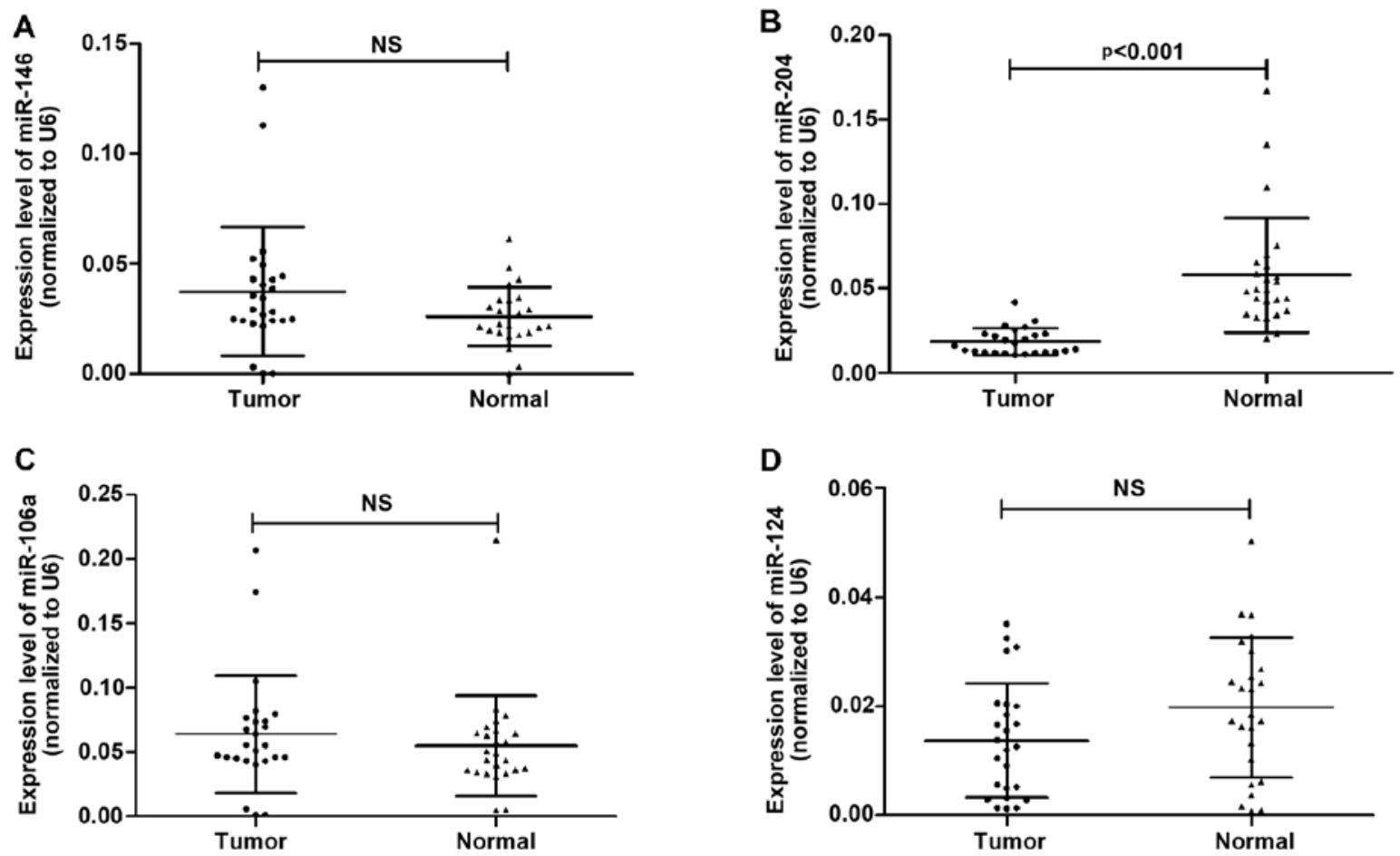

Figure 1. Expression level of target miRNAs in the plasma of patients with non-small cell lung cancer (NSCLC) and healthy controls in the training phase. Expression levels of miRNAs are presented as the means \pm SD. (A) miR-146. (B) miR-204. (C) miR-106a. (D) miR-124. NS, no significance.
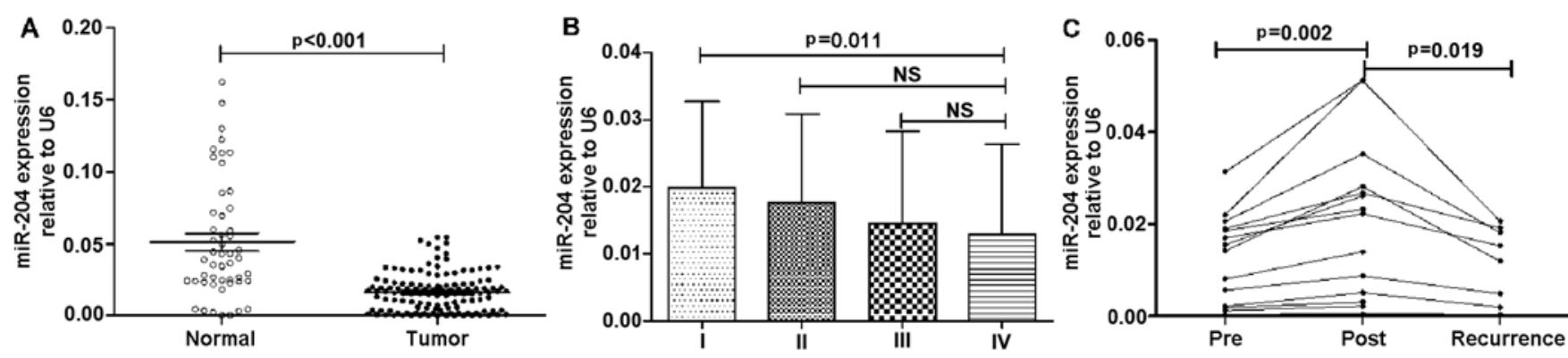

Figure 2. Expression levels of miR-204 in plasma samples in the validation phase. (A) The miR-204 expression levels in plasma were significantly higher in patients with non-small cell lung cancer (NSCLC) $(n=126)$ than in the healthy controls $(n=50)(p<0.001)$. (B) The miR-204 expression level was significantly decreased in patients with stage IV of the disease when compared with that in patients with stage I of the disease, $p=0.011$. (C) The miR-204 expression levels were significantly higher in plasma obtained post-operatively than those in plasma obtained pre-operatively $(\mathrm{p}=0.002)$, and in 8 cases, the plasma miR-204 levels were re-downregulated when patients developed post-operative recurrence $(\mathrm{p}=0.019)$. RNU6B (U6) was used as an internal control. NS, no significance.
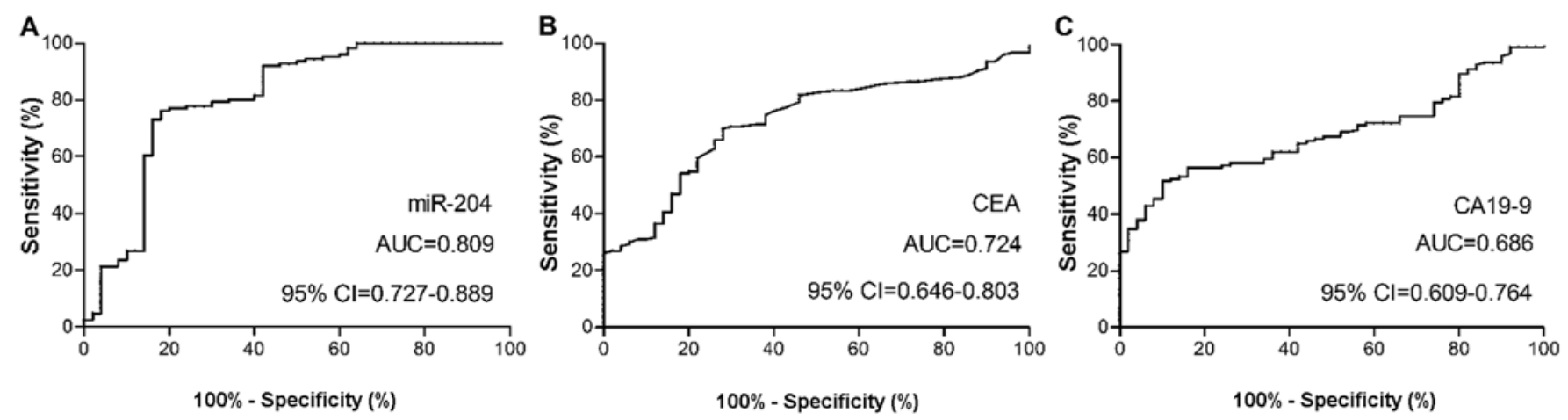

Figure 3. Comparison of the receiver operating characteristic (ROC) curves for (A) miR-204 in plasma and the conventional non-small cell lung cancer (NSCLC) markers, (B) carcinoembryonic antigen (CEA) and (C) carbohydrate antigen 19-9 (CA19-9) in serum.

Expression of miR-204 in plasma samples in relation to prognosis. The patients were divided into 2 groups according to the median relative expression levels of miR-204: the low and high expression group. As shown in Fig. 4, the overall survival (OS) 

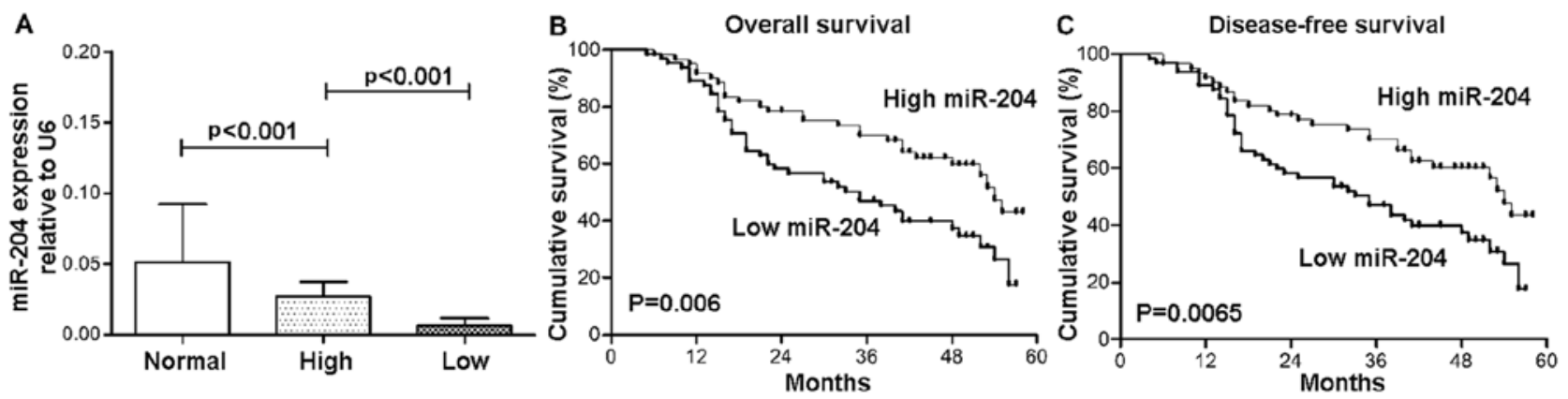

Figure 4. Survival analysis of patients with non-small cell lung cancer (NSCLC) grouped by the median level of miR-204. (A) The levels of miR-204 of the high-expression group were significantly lower than that of controls, while higher than that of the low-expression group. (B and C) Kaplan-Meier analysis showed that in patients with NSCLC with higher miR-204 expression in the plasma, this correlated with a poor overall survival ( $\mathrm{p}=0.006)$ and shorter disease-free survival $(\mathrm{p}=0.0065)$.

Table II. Patient clinicopathological characteristics and correlation with plasma miR-204 expression levels.

\begin{tabular}{|c|c|c|c|}
\hline Characteristics & $\begin{array}{c}\text { No. of } \\
\text { patients } \\
n=126\end{array}$ & $\begin{array}{c}\text { miR-204 } \\
\text { expression } \\
(\text { means } \pm \text { SD) }\end{array}$ & p-value \\
\hline
\end{tabular}

Gender
Female
Male
Age (years)
$<60$
$\geq 60$

$0.346^{\mathrm{a}}$

Smoking condition

Former/never

Current

$\begin{array}{ll}45 & 0.015 \pm 0.012 \\ 81 & 0.017 \pm 0.014\end{array}$

$81 \quad 0.017 \pm 0.014$

Histological type

Adenocarcinoma

Squamous cell carcinoma

Other

$\begin{array}{ll}61 & 0.018 \pm 0.014 \\ 65 & 0.015 \pm 0.013\end{array}$

$0.186^{\mathrm{a}}$

Differentiation

Well/moderate

Poor

$47 \quad 0.017 \pm 0.013$

$79 \quad 0.016 \pm 0.014$

TNM stage

$$
\text { I, III }
$$

$63 \quad 0.017 \pm 0.013$

$53 \quad 0.015 \pm 0.014$

$10 \quad 0.019 \pm 0.011$

$0.858^{\mathrm{a}}$

$0.359^{\mathrm{b}}$

$86 \quad 0.017 \pm 0.013$

$40 \quad 0.014 \pm 0.014$

$0.157^{\mathrm{a}}$

Lymph node metastasis

N0

N1-3

$66 \quad 0.019 \pm 0.013$

$60 \quad 0.014 \pm 0.014$

$77 \quad 0.017 \pm 0.013$

$49 \quad 0.016 \pm 0.014$

Distant metastasis

No

$97 \quad 0.017 \pm 0.013$

Yes

$29 \quad 0.013 \pm 0.014$

Recurrence

$\begin{array}{lrr}\text { No } & 111 & 0.017 \pm 0.014 \\ \text { Yes } & 15 & 0.012 \pm 0.010\end{array}$

${ }^{\mathrm{a}}$ Mann-Whitney test; ${ }^{\mathrm{b}}$ Kruskal-Wallis test; SD, standard deviation; former, stopped smoking for over 2 years; never, no smoking history; current, current smoker; TNM, tumor-node metastasis.

$0.009^{\mathrm{a}}$

$0.432^{\mathrm{a}}$ and disease-free survival (DFS) of the patients with lower plasma levels of miR-204 were poorer than those of patients with higher levels of miR-204 [lower (median survival $=32$ months) vs. higher (median survival $=43$ months) for $\mathrm{OS}(\mathrm{p}=0.006)$, and lower (30 months) vs. higher (40 months) for DFS ( $\mathrm{p}=0.0065)$, respectively].

We also carried out univariate and multivariate analyses in order to examine the prognostic significance of the clinicopathological characteristics and miR-204 expression. Using Cox regression analysis, we found that a higher TNM stage (III and IV), positivity for distant metastasis, recurrence and a low miR-204 expression significantly correlated with a relative risk of mortality of 4.311, 12.942, 2.757 and 1.936, respectively (Table III). All these prognostic factors were confirmed by multivariate analysis. These data revealed that the lower expression of miR-204 in plasma [hazard ratio (HR), 1.712; 95\% confidence interval (CI), 1.038-2.825; $\mathrm{p}=0.035]$, a higher TNM stage (HR, 2.215; 95\% CI, 1.207-4.063; $\mathrm{p}=0.010$ ), the presence of distant metastasis (HR, 8.086; 95\% CI, 4.176-15.657; $\mathrm{p}=0.000$ ) and recurrence (HR, 2.329; 95\% CI, 1.251-4.334; $\mathrm{p}=0.008$ ) were independently associated with a significantly increased risk of mortality (Table III).

\section{Discussion}

miRNAs are generally defined as regulatory molecules, and studies have indicated that the abnormal expression of miRNAs is associated with various types of cancer. More importantly, miRNAs have been found to be stably expressed in bodily fluids, including serum, plasma, urine, and saliva (10,27-30), thus providing a promising strategy for the identification of miRNAs for use as a less invasive and more convenient tool that can be used in diagnosis, prognosis or treatment monitoring. However, the majority of studies have focused on the examination of miRNA levels in tissue specimens (7), and only a limited number have examined the practicability of plasma miRNAs as biomarkers $(13,14)$. As is already known, early detection is the most effective method of reducing the high mortality rate of patients with NSCLC. Thus, in this study, we investigated potential miRNAs for use as biomarkers for the diagnosis of NSCLC.

In the present study, we initially set out to analyze the plasma levels of 4 miRNAs, including miR-124, miR-204, miR-106a and miR-146 in patients with NSCLC and healthy controls 
Table III. Cox proportional regression analysis for assessing the correlation of miR-204 expression with the prognosis of patients with non-small cell lung cancer.

\begin{tabular}{|c|c|c|c|c|c|c|}
\hline \multirow[b]{2}{*}{ Variables } & \multicolumn{3}{|c|}{ Univariate analysis } & \multicolumn{3}{|c|}{ Multivariate analysis } \\
\hline & HR & $95 \% \mathrm{CI}$ & $\mathrm{p}$-value & $\mathrm{HR}$ & $95 \% \mathrm{CI}$ & p-value \\
\hline Age $(<60, \geq 60)$ & 0.962 & $0.602-1.539$ & 0.872 & & & \\
\hline Gender (fema) & 1.106 & $0.689-1.775$ & 0.678 & & & \\
\hline Distant metastasis (no, yes)le, male) & 1.006 & $0.611-1.659$ & 0.980 & & & \\
\hline Smoking history (former/never, current) & 1.095 & $0.667-1.797$ & 0.720 & & & \\
\hline Histological type (AC, SCC) & 1.253 & $0.880-1.786$ & 0.211 & & & \\
\hline Differentiation (well/moderate, poor) & 1.138 & $0.676-1.716$ & 0.805 & & & \\
\hline TNM stage (I/II, III/IV) & 4.311 & $2.575-7.219$ & 0.000 & 2.215 & $1.207-4.063$ & 0.010 \\
\hline Lymph node metastasis (N0, N1-3 & 12.942 & $7.351-22.788$ & 0.000 & 8.086 & $4.176-15.657$ & 0.000 \\
\hline Recurrence (no, yes) & 2.757 & $1.490-5.100$ & 0.001 & 2.329 & $1.251-4.334$ & 0.008 \\
\hline miR-204 level (high, low) & 1.936 & $1.193-3.143$ & 0.008 & 1.712 & $1.038-2.825$ & 0.035 \\
\hline
\end{tabular}

AC, adenocarcinoma; SCC, squamous cell carcinoma; TNM, tumor-node metastasis; HR, hazard ratio; CI, confidence interval.

based on 50 participants (training phase). These miRNAs have been reported to act as oncogenes or tumor-suppressors, and have been shown to be associated with several critical biological processes, including migration, invasion, apoptosis and proliferation $(5,6)$. However, these selected miRNAs exhibited no significant differences in expression between the patients with NSCLC and the healthy controls, with the exception of miR-204. Thus, we subsequently measured the expression levels of miR-204 in plasma samples from a large cohort of patients with NSCLC and evaluated the diagnostic capabilities and prognostic value of miR-204 in these patients. To the best of our knowledge, this study is the first study which used plasma samples to evaluate the predictive value of miR-204 as a potential biomarker for NSCLC.

As a tumor suppressor, miR-204 has been reported to be markedly decreased in malignant tissues, including gastric cancer (16), breast cancer (17), renal cell carcinoma (18), and NSCLC (19). Researchers firstly identified miR-204 as a novel, non-invasive biomarker for endometrioid endometrial cancer diagnosis by using genome-wide serum miRNA expression profiling (31). In the present study, we also noted a significantly decreased level of miR-204 in plasma from patients with NSCLC compared with healthy controls. Our findings were consistent with those of previous studies and suggested that the downregulation of miR-204 is involved in the initiation and progression of NSCLC. Functionally, the loss of miR-204 promotes cancer cell migration and invasion by regulating target genes $(19,20)$. miR-204 plays a role in the direct regulation of epithelial-to-mesenchymal transition (EMT) through its targeting of SMAD4 (32). The overexpression of miR-204 has been shown to inhibit cancer cell proliferation in gastric and colorectal cancer $(21,33)$. Thus, the findings of these abovementioned previous studies, as well as those from our study support the tumor-suppressor role of miR-204 in cancer.

Certain serum markers have been widely used in the diagnosis of cancer, such as CEA and CA19-9. Therefore, we compared the diagnostic capabilities of miR-204 with those of conventional tumor markers. Based on ROC curve analysis, we found that miR-204 was suitable for differentiating patients with NSCLC from healthy individuals, with a sensitivity and specificity of 76 and $82 \%$, respectively. The AUC value for plasma miR-204 was 0.809, which was higher than the AUC values obtained with CEA and CA19-9. Our results suggest that plasma miR-204 is a good diagnostic marker and a more reliable alternative for the diagnosis of NSCLC.

miR-204 has previously been shown to be downregulated in tissue specimens from patients with $\operatorname{NSCLC~}(19,34)$. However, whether plasma levels of miR-204 are associated with prognosis, OS and DFS in patients with NSCLC remains unclear. In this study, we found that the plasma levels of miR-204 were significantly upregulated in plasma collected post-operatively compared to that in plasma collected pre-operatively from the same patients, and was then downregulated again in post-operative recurrent cases. Our results suggest that miR-204 may be used to monitor and evaluate the tumor dynamics of NSCLC. Moreover, we observed a stepwise decreased expression of plasma miR-204 with the increasing tumor stage. In addition, we analyzed the prognostic role of the expression of miR-204 in plasmas from patients with NSCLC. We found that a low expression of miR-204 in plasma samples correlated with an advanced clinical stage and distant metastasis in NSCLC. This result was in accordance with that of previous studies $n$ breast cancer (17), nasopharyngeal carcinoma (35) and endometrial cancer (36). These results suggest that miR-204 in plasma isa potential marker which reflects cancer progression.

It has previously been reported that the low expression of miR-204 correlates with a poor prognosis in several types of cancer $(17,19,21,37)$. Thus, in the present study we further analyzed the association of plasma levels of miR-204 with patient clinical outcomes. Similarly, we found that low plasma levels of miR-204 correlated with poorer survival by using the log-rank test, and univariate and multivariate analyses, indicating that miR-204 is an independent prognostic factor for survival. These are surprising novel data which are important for our current understanding of NSCLC. Moreover, Yin et al found that restoring miR-204 expression promoted tumor sensitivity to chemotherapy in colorectal cancer patients (21). miR-204 targeted Bcl-2 messenger RNA and increased the 
responsiveness of gastric cancer cells to 5-fluorouracil and oxaliplatin treatment (38). If these findings are further determined in NSCLC patients, miR-204 may thus have the potential to assist in the treatment of patients with NSCLC and reduce the mortality rate in patients with this disease.

In conclusion, in the present study, we demonstrated that miR-204 in plasma from patients with NSCLC was downregulated. Thus, we suggest that the plasma levels of miR-204 are more reliable than the levels of CEA and CA19-9 for diagnosis. More importantly, the decreased expression of miR-204 in plasma is likely a promising marker for predicting poor survival in patients with NSCLC. Further investigations with larger sample sizes and other types of cancer are warranted be in order to evaluate the possibility of using plasma miR-204 as a non-invasive marker for cancer detection and clinical outcome prediction.

\section{References}

1. Jemal A, Siegel R, Ward E, Hao Y, Xu J and Thun MJ: Cancer statistics, 2009. CA Cancer J Clin 59: 225-249, 2009.

2. Siegel R, Naishadham D and Jemal A: Cancer statistics, 2013. CA Cancer J Clin 63: 11-30, 2013.

3. Wu YL and Zhou Q: Clinical trials and biomarker research on lung cancer in China. Expert Opin Ther Targets 16 (Suppl 1): S45-S50, 2012.

4. Dykxhoorn DM: MicroRNAs and metastasis: little RNAs go a long way. Cancer Res 70: 6401-6406, 2010.

5. Garzon R, Fabbri M, Cimmino A, Calin GA and Croce CM: MicroRNA expression and function in cancer. Trends Mol Med 12: 580-587, 2006.

6. Zhang B, Pan X, Cobb GP and Anderson TA: microRNAs as oncogenes and tumor suppressors. Dev Biol 302: 1-12, 2007.

7. Li W, Wang Y, Zhang Q, Tang L, Liu X, Dai Y, Xiao L, Huang S, Chen L, Guo Z, Lu J and Yuan K: MicroRNA-486 as a biomarker for early diagnosis and recurrence of non-small cell lung cancer. PLoS One 10: e0134220, 2015.

8. Mavridis K, Gueugnon F, Petit-Courty A, Courty Y, Barascu A, Guyetant S and Scorilas A: The oncomiR miR-197 is a novel prognostic indicator for non-small cell lung cancer patients. Br J Cancer 112: 1527-1535, 2015.

9. Tejero R, Navarro A, Campayo M, Viñolas N, Marrades RM, Cordeiro A, Ruíz-Martínez M, Santasusagna S, Molins L, Ramirez J and Monzó M: miR-141 and miR-200c as markers of overall survival in early stage non-small cell lung cancer adenocarcinoma. PLoS One 9: e101899, 2014.

10. Mitchell PS, Parkin RK, Kroh EM, Fritz BR, Wyman SK, Pogosova-Agadjanyan EL, Peterson A, Noteboom J, O'Briant KC, Allen A, et al: Circulating microRNAs as stable blood-based markers for cancer detection. Proc Natl Acad Sci USA 105: 10513-10518, 2008.

11. Patnaik SK, Mallick R and Yendamuri S: Detection of microRNAs in dried serum blots. Anal Biochem 407: 147-149, 2010.

12. Yang $X$, Zeng Z, Hou Y, Yuan T, Gao C, Jia W, Yi X and Liu M: MicroRNA-92a as a potential biomarker in diagnosis of colorectal cancer: a systematic review and meta-analysis. PLoS One 9: e88745, 2014.

13. Shen J, Hruby GW, McKiernan JM, Gurvich I, Lipsky MJ, Benson MC and Santella RM: Dysregulation of circulating microRNAs and prediction of aggressive prostate cancer Prostate 72: 1469-1477, 2012

14. Ng EK, Li R, Shin VY, Jin HC, Leung CP, Ma ES, Pang R, Chua D, Chu KM, Law WL, et al: Circulating microRNAs as specific biomarkers for breast cancer detection. PLoS One 8: e53141, 2013.

15. Gorur A, Balci Fidanci S, Dogruer Unal N, Ayaz L, Akbayir S, Yildirim Yaroglu H, Dirlik M, Serin MS and Tamer L: Determination of plasma microRNA for early detection of gastric cancer. Mol Biol Rep 40: 2091-2096, 2013.

16. Zhang B, Yin Y, Hu Y, Zhang J, Bian Z, Song M, Hua D and Huang Z: MicroRNA-204-5p inhibits gastric cancer cell proliferation by downregulating USP47 and RAB22A. Med Oncol 32: 331, 2015.

17. Li W, Jin X, Zhang Q, Zhang G, Deng X and Ma L: Decreased expression of miR-204 is associated with poor prognosis in patients with breast cancer. Int J Clin Exp Pathol 7: 3287-3292, 2014.
18. Munari E, Marchionni L, Chitre A, Hayashi M, Martignoni G, Brunelli M, Gobbo S, Argani P, Allaf M, Hoque MO and Netto GJ: Clear cell papillary renal cell carcinoma: micro-RNA expression profiling and comparison with clear cell renal cell carcinoma and papillary renal cell carcinoma. Hum Pathol 45: $1130-1138,2014$

19. Shi L, Zhang B, Sun X, Lu S, Liu Z, Liu Y, Li H, Wang L, Wang X and Zhao C: MiR-204 inhibits human NSCLC metastasis through suppression of NUAK1. Br J Cancer 111: 2316-2327, 2014.

20. Imam JS, Plyler JR, Bansal H, Prajapati S, Bansal S, Rebeles J, Chen HI, Chang YF, Panneerdoss S, Zoghi B, et al: Genomic loss of tumor suppressor miRNA-204 promotes cancer cell migration and invasion by activating AKT/mTOR/Rac1 signaling and actin reorganization. PLoS One 7: e52397, 2012.

21. Yin Y, Zhang B, Wang W, Fei B, Quan C, Zhang J, Song M, Bian Z, Wang Q, Ni S, et al: miR-204-5p inhibits proliferation and invasion and enhances chemotherapeutic sensitivity of colorectal cancer cells by downregulating RAB22A. Clin Cancer Res 20: 6187-6199, 2014.

22. Wu X, Zeng Y, Wu S, Zhong J, Wang Y and Xu J: MiR-204, down-regulated in retinoblastoma, regulates proliferation and invasion of human retinoblastoma cells by targeting CyclinD2 and MMP-9. FEBS Lett 589: 645-650, 2015.

23. Li G, Luna C, Qiu J, Epstein DL and Gonzalez P: Role of miR-204 in the regulation of apoptosis, endoplasmic reticulum stress response, and inflammation in human trabecular meshwork cells. Invest Ophthalmol Vis Sci 52: 2999-3007, 2011.

24. Livak KJ and Schmittgen TD: Analysis of relative gene expression data using real-time quantitative PCR and the 2(-Delta Delta C(T)) Method. Methods 25: 402-408, 2001.

25. Niklinski J, Furman M, Laudanski J and Kozlowski M: Prognostic value of pretreatment CEA, SCC-Ag and CA 19-9 levels in sera of patients with non-small cell lung cancer. Eur J Cancer Prev 1: 401-406, 1992.

26. Ruopp MD, Perkins NJ, Whitcomb BW and Schisterman EF: Youden Index and optimal cut-point estimated from observations affected by a lower limit of detection. Biom J 50: 419-430, 2008.

27. Schwarzenbach H, Nishida N, Calin GA and Pantel K: Clinical relevance of circulating cell-free microRNAs in cancer. Nat Rev Clin Oncol 11: 145-156, 2014.

28. Kang K, Peng X, Luo J and Gou D: Identification of circulating miRNA biomarkers based on global quantitative real-time PCR profiling. J Anim Sci Biotechnol 3: 4, 2012.

29. Wang J, Zhang KY, Liu SM and Sen S: Tumor-associated circulating microRNAs as biomarkers of cancer. Molecules 19: 1912-1938, 2014.

30. Cortez MA, Bueso-Ramos C, Ferdin J, Lopez-Berestein G, Sood AK and Calin GA: MicroRNAs in body fluids - the mix of hormones and biomarkers. Nat Rev Clin Oncol 8: 467-477, 2011.

31. Jia W, Wu Y, Zhang Q, Gao G, Zhang C and Xiang Y: Identification of four serum microRNAs from a genome-wide serum microRNA expression profile as potential non-invasive biomarkers for endometrioid endometrial cancer. Oncol Lett 6: 261-267, 2013.

32. Wang Y, Li W, Zang X, Chen N, Liu T, Tsonis PA and Huang Y: MicroRNA-204-5p regulates epithelial-to-mesenchymal transition during human posterior capsule opacification by targeting SMAD4. Invest Ophthalmol Vis Sci 54: 323-332, 2013.

33. Zhou X, Li L, Su J and Zhang G: Decreased miR-204 in H.pyloriassociated gastric cancer promotes cancer cell proliferation and invasion by targeting SOX4. PLoS One 9: e101457, 2014.

34. Xia Y, Zhu Y, Ma T, Pan C, Wang J, He Z, Li Z, Qi X and Chen Y: miR-204 functions as a tumor suppressor by regulating SIX1 in NSCLC. FEBS Lett 588: 3703-3712, 2014.

35. Ma L, Deng X, Wu M, Zhang G and Huang J: Down-regulation of miRNA-204 by LMP-1 enhances CDC42 activity and facilitates invasion of EBV-associated nasopharyngeal carcinoma cells. FEBS Lett 588: 1562-1570, 2014.

36. Bao W, Wang HH, Tian FJ, He XY, Qiu MT, Wang JY, Zhang HJ, Wang LH and Wan XP: A TrkB-STAT3-miR-204-5p regulatory circuitry controls proliferation and invasion of endometrial carcinoma cells. Mol Cancer 12: 155, 2013.

37. Ryan J, Tivnan A, Fay J, Bryan K, Meehan M, Creevey L, Lynch J, Bray IM, O'Meara A, Tracey L, et al: MicroRNA-204 increases sensitivity of neuroblastoma cells to cisplatin and is associated with a favourable clinical outcome. Br J Cancer 107: 967-976, 2012.

38. Sacconi A, Biagioni F, Canu V, Mori F, Di Benedetto A, Lorenzon L, Ercolani C, Di Agostino S, Cambria AM, Germoni S, et al: miR-204 targets Bcl-2 expression and enhances responsiveness of gastric cancer. Cell Death Dis 3: e423, 2012. 